\title{
From consultation to participation in public health research: reflections on a community-based research partnership
}

\author{
Lauren J Breen ${ }^{*}$ and Moira O'Connor
}

\begin{abstract}
Background: Road traffic crashes and their outcomes are substantial global public health issues and public health initiatives are increasingly involving relevant community members in order to create sustainable change. This paper describes an applied research project utilizing participatory methods to establish a road trauma support service in Western Australia and reflects on the extent of participation in the community-based research partnership. Community-based participatory research (CBPR) provided the basis for the research project conducted in partnership with 34 government and non-government agency representatives and people affected personally by road trauma and which resulted in 22 recommendations for establishing the service.
\end{abstract}

Findings: Attempts to position the group as co-researchers highlighted the dynamic interplay of factors that hinder and enable participation in participatory research. Barriers to participation within the research process included the limited time and funds, reluctance to share authorship, and a lack of clarity regarding roles and processes. Factors that enabled participation were the recognition of each member's expertise, providing different forms and methods of communication, and the reimbursement of costs according to role.

Discussion: In May 2012, the Government of Western Australia announced it would fund the recommendations and Road Trauma Support Western Australia was launched in November 2013. Notwithstanding this successful outcome, there were varied experiences of participation in the research process, and this was despite the use of a research methodology that is by definition participatory, with explicit and embedded participatory structures and processes. The research project shows that elements of CBPR can be incorporated into public health research, even in projects with externally-imposed time and budget constraints.

Keywords: Participatory action research, Community-based participatory research, Trauma, Injury, Bereavement, Consumer involvement

\section{Background}

Road traffic crashes result in the deaths of approximately 1.24 million and injuries to 20 to 50 million people every year [1]. Globally, traffic crashes are the eighth leading cause of death and the leading cause of death for people aged 15 to 29 years [2]. In 1974, the World Health Assembly declared traffic crashes to be a substantial public health problem and the increasing scope of the issue prompted the World Health Organization to dedicate its annual World Health Day in 2004 to road safety [3].

\footnotetext{
* Correspondence: lauren.breen@curtin.edu.au

School of Psychology and Speech Pathology, Faculty of Health Sciences, Curtin University, GPO Box U1987, Perth 6845, Western Australia
}

Crashes and their consequences affect drivers, riders, and passengers; their family, friends, and colleagues; witnesses and emergency service workers; and members of the wider community. Outcomes include serious physical injuries and temporary or permanent disability $[4,5]$, intense distress and grief [6,7], post-trauma reactions and psychiatric disorders [8,9], social isolation and stigma $[10,11]$, decreases in quality of life [12], carer burden $[13,14]$, and considerable financial costs $[15,16]$. Notwithstanding the frequency of crash injuries and fatalities and the differential risk borne by vulnerable road user groups [17], the people affected by these outcomes are neglected [3]; this is despite post-crash care being positioned as one of five pillars of road safety, alongside 
road safety management, safer roads, safer vehicles, and safer road users [1].

Despite these consequences, the focus of traffic crash prevention and management has traditionally been on the 'hardware' aspects such as road engineering, vehicle safety, and punitive behavioral measures (e.g., licensing, fines) to shape road user behavior [1,3]. In contrast, traffic crash consequences are often 'invisible' to service providers and the wider community [10,11]. Furthermore, research has highlighted the disconnect between government rhetoric, which draws on a community discourse to emphasize road safety as a community responsibility, with practices that discourage community narratives from being voiced, by regulating "who is involved, how often they are involved, and the roles of those involved" ([10], p. 47). Over the past few decades, several countries have seen the emergence of grassroots community groups advocating on behalf of traffic victims (e.g., Mothers Against Drunk Driving in the United States; Fédération Européene des Victimes de al Route/ Federation of European Road Traffic Victims). While these groups differ in their origins, resources, and objectives, many were established by people who are injured or bereaved as a result of crashes. Together, these groups organize events for memorial and remembrance purposes (e.g., the annual World Day of Remembrance for Road Traffic Victims), develop and distribute brochures and other materials, many of which contain stories of road traffic victims and their families; campaign for improved road infrastructure and crash prevention; and advocate for improved post-crash responses, with a focus on emergency care as well as social, medical and legal services [18].

It is increasingly recognized that mainstream research methods, which are led and owned by researchers as experts and rarely give any consideration to context, are often ill-suited to the investigation of complex health problems [19]. Alongside this, there has been an increasing awareness of the importance of communities being actively engaged in research to identify, define, and discover ways to resolve or solve the health problems affecting them [20-22]. These developments underpin the increasing use of participatory research methods that promote the involvement relevant community members in order to address health issues, resolve disparities, and create sustainable change $[23,24]$.

Community-based participatory research (CBPR) is an overarching term used to describe research a collection of research approaches variously described with monikers such as action research, participatory action research, and cooperative inquiry [25]. There are three defining features of CBPR - the research occurs with exploited/oppressed communities rather than on them [26]; the research addresses the specific concerns of a community to achieve change, often in relation to health disparities [27-30]; and the research involves a research and action process [24]. The co-researchers are typically involved in decisions about all stages of the research [31]. However, CBPR research has its own challenges, notably the extent to which the process is participatory. Typically, explicit accounts of initiating and sustaining participation are not described in published papers and the challenges of promoting participation tend to be oversimplified or obscured [32,33].

\section{Methods}

This project emerged from an identified need in the community. A small group of people bereaved through traffic crashes had been active in advocating for a road trauma support service in Western Australia since 2000 but, until recently, their campaign for a structured support service for people affected by road trauma had not been successful (see [10], for a discussion of this action). The absence of a dedicated road trauma service was especially problematic, given that Western Australia consistently demonstrates the highest traffic crash fatality rate of all Australian states [34]. Further, there are considerable gaps and limitations in the current services available in Western Australia, which are difficult to identify, costly to access, limited due to time restrictions or staffing resources, and available only in certain regions rather than state-wide.

Due to these gaps, in mid-2010, the Western Australian Department of Health commissioned expressions of interest from researchers to investigate mechanisms and associated costs and to make recommendations in regard to establishing a road trauma support service in Western Australia to provide sustainable peer support and professional counseling for road trauma victims, family members, witnesses and others who are adversely affected by road trauma events. Although the timeline and general aims were established by the Department of Health, there was greater leeway in refining the aims are determining the ways in which they would be addressed. In proposing our research plan (which was ultimately funded), we drew upon CBPR to privilege the perspectives of people working with, or affected by, road trauma with a goal of producing health service change.

Following approval from the Curtin University Human Research Ethics Committee (approval number HR29/ 2011; in compliance with the Helsinki Declaration), a stakeholder reference group was formed, comprising 34 representatives from government (e.g., police, hospitals, victim support, emergency services, coroner's office) and non-government agencies (e.g., carer and disability advocacy, injury control, a road user group, the peak body for support groups), as well as community members bereaved by and/or injured in traffic crashes. Several group members already knew each other due to their ongoing 
advocacy for road trauma support and/or involvement in previous initiatives (e.g., the development of young driver training policy, the unveiling of a crash fatality remembrance memorial). Face-to-face meetings were complemented by regular, ongoing contact via email, post, and telephone.

The group decided to examine existing road trauma support programs in Australia in order to determine the parameters of a similar service in Western Australia and assisted in the development of a semi-structured interview protocol. Using this protocol, we conducted telephone interviews with key personnel at each service and these were complemented by the analysis of each service's websites, mission statements, annual reports, brochures, and evaluations. Attention was paid to the history and development of each service; promotion of services to service users and referring professionals; referral pathways to and from each service; fees; scope and duration of service delivery; recruitment, training, and appointment of paid and volunteer staff; resources required, including accommodation, administration, computing, communications and promotional materials; metropolitan and regional service delivery; establishment and ongoing annual costs and funding sources; and service evaluation. We wrote case study summaries of the services.

The group used the summaries to determine which services would be visited by the first author (the project's budget would not cover visits to all services or the travel of more than one person), and finalize the data collection strategy. Three services - those in the states of Victoria, South Australia, and Tasmania - were chosen for visits because they were considered to offer the most comprehensive road trauma services. Using the protocol described earlier, the first author conducted semistructured, face-to-face interviews of approximately one hour each with key staff members (i.e., counselors, managers, executive officers, volunteer coordinators) from the three services in order to expand on the information gleaned from the case studies. Additionally, the travel to each service facilitated observations of each service's location and resources (e.g., office space, number of staff, access via public transport, and parking). This analysis of existing services elsewhere in Australia revealed each service's strengths and limitations, which informed the recommendations for establishing the road trauma support service in Western Australia.

We wrote a draft report and disseminated it within the group for comments and changes and made every effort to incorporate them into the final report. Once complete, the final report [35] was made available (in hard and electronic copy) to each group member, the visited services, and to the Department of Health. The report included 22 recommendations for the establishment of the road trauma support service. The findings and recommendations have been published previously [36]. What follows is an overview of the research process and the lessons learned in attempting to optimize the participation of government and non-government agency representatives and community members affected by road trauma in the research process.

\section{Findings}

In conducting this research, the tension between the ideal and reality of participation became apparent. Ultimately, this tension was reinforced by power differentials between the researchers and the group, and between group members, and several challenges arose as a result. These challenges encountered in attempting to optimize participation, as well as the facilitators of participation, are discussed below. Articulating these challenges allowed us to implement strategies to mitigate their impact, leading to an overall successful outcome.

\section{Challenges to participation}

First, the development of co-researcher partnerships between group members and between the university researchers and co-researchers was limited by time and budgetary constraints. Our ability to develop a true partnership with the group while completing the contracted objectives of the project was complicated by the tight timeline required by the funding body. We negotiated a three-month extension to the project, and while this extension helped, the process adopted did not engender full participation from the outset. Instead, it began as a consultative process and developed in its participatory nature over time, a common distinction in participatory research [37]. Additional time would have allowed more discussion about the scope of the study, allowing the group to provide further input. Inclement weather prevented the majority of group members from being present at one face-to-face meeting, meaning that the majority of members were not there to participate in the decision-making discussion. Unfortunately, the project's timeline also precluded rescheduling the meeting and the authors communicated via email and telephone with group members who were not able to attend. Additionally, we would have preferred the group to nominate a member to accompany the first author on the visits to existing services elsewhere in Australia. Such visits would have been invaluable to the service providers and to the community members as well as an indication of the coresearcher status we were attempting to foster. However, this was not possible due to the budget limitations.

Second, surprisingly, we encountered hesitancy to share authorship among group members. One group member expressed discomfort concerning sections of the draft report that implied the recommendations were supported 
by the group members' organizations (where applicable). This member explained that the report would need to be endorsed through each organization's board of management for the report to state or imply as such. This perspective was opposed by other group members who expressed disappointment and anger and inferred that the particular organization was against the proposed funding model from the beginning. However, the time restrictions on the project meant that we would be unable to gather the various permissions for everyone to author the report so we decided that the authorship had to be separated from participation in the group. Duckett and Fryer [27] wrote about separating the research process from the publication, so they authored publications without the coresearchers because the co-researchers wished to remain anonymous. We reworded relevant sections of the draft and asked the group about their suggestions for alternate wording and confirmed their satisfaction with the changes. One member emailed that she was satisfied with the changes and appreciated the participatory approach taken to getting feedback on the draft:

It is difficult to manage a large and diverse group under any circumstance let alone when it involves a highly emotional and personally relevant topic. I am grateful of your consideration of my feedback and appreciate that you have accommodated that.

Third, despite our attempts to communicate clearly, at times there appeared to be a lack of understanding about the processes necessary in research. Ultimately, the Department of Health was the client, which weakened our attempts to promote the group's ownership of the study. For example, some group members expressed frustration at the time it took to complete the project and informed a member of the opposing political party. This politician emailed an author to inquire about the progress of the study and the anticipated completion date. The author replied by suggesting that the member contact the Department of Health and/or the Minister for Health. However, the politician and some members of the stakeholder reference group described the reply as being obstructive as they did not understand that ethically it was not appropriate to provide information to third parties.

As further example, the university's public relations section organized a media campaign wherein articles relating to the research appeared on the university homepage, a science network website, and in various newspapers. We asked that the group, or at least some members, be featured in the newspaper articles and accompanying photographs but it became clear that the media interest was based on a university-generated press release and the newspapers' journalists stated they were not interested in featuring group members. Notwithstanding these issues, it was serendipitous that there was little conflict because, given the time constraints, we did not develop a group protocol or terms of reference document. Clarity concerning role and processes is typically essential in outlining the purpose and objectives of the research project and the membership and governance of the group [19].

\section{Facilitators of participation}

In order to promote participation and mitigate tokenism, we first aimed to include an approximately equal number of representatives from services and people with personal experiences of traffic crash consequences and, throughout the project, we described both types of representatives as experts - one with expertise in service delivery and the other with expertise from personal experience. The inclusion of the community members meant that the issues under discussion were related to 'real-life' experiences, situations, and realities rather than abstractions [38]. For instance, in the third meeting, a service representative suggested that current services were adequate and there was no need for a comprehensive road trauma support service. Three community representatives (one who had been injured and two who were bereaved) each shared some of their experiences of attempting to negotiate services, which highlighted the 'real-world' gaps in the current system. Additionally, in the report, we acknowledged each member by name in alphabetical order and without distinction between the service and community representatives.

Second, we were acutely aware of the differences in the ability of individual group members to participate in the research and used several methods of communication to mitigate these differences. For instance, while the service representatives had ready access to email, fax, and printing facilities, this was sometimes not the case for the community representatives. In recognition of these differences, all group members had the option of receiving information via email or via post. Many community representatives requested that documents were posted as they did not have facilities to print them. Radermacher and Sonn [39] described the different strategies they used in disseminating information to different group members to maximize access and participation. However, they reported that their attempts to use different methods of communication still had a deleterious effect on the ability of some members to participate fully.

Third, we were aware that service representatives were paid by their employers for their participation while the community representatives were representing themselves in an unpaid capacity. As such, the members with personal experiences of traffic crash consequences each received a shopping voucher in recognition of their time and associated expenses such as transport and parking. Together, we believe these strategies lessened the impact of 
the challenges. Although we did not formally gather feedback from the group (e.g., through an anonymous questionnaire) on their perceptions of participation throughout the research process, spontaneous feedback from group members provide some evidence of support for our reflections. One member emailed:

We value the work carried out by the dedicated investigators who demonstrated both compassion and professionalism throughout the research process... Importantly, the project was based on the contributions from the individuals and families who have campaigned for many years for a road trauma support service in Western Australia. The project has also taken into account key human rights principles of self determination, partnerships in research and inclusion within society.

\section{Discussion}

The destabilization of power hierarchies and the creation and maintenance of equitable power structures are major challenges of CBPR and were challenges we faced at times throughout the study. Typically, discussions about unequal power focus on the difference between academic researchers and the co-researchers [31,32]. In this study, however, the differences between the community members and the service representatives were just as prominent as those between the group and the researchers. These tensions were acknowledged and sometimes compromises were made. Despite attempts to include the stakeholder reference group as equal co-researchers, the process, at least at first, would best be described as consultative. However, over time, the process became increasingly participatory.

The progression from participants to co-researchers was evident in the group members' comments on the draft report. Despite the length of the draft (approximately 32,000 words) and the deadline for comments (about three weeks), all members of the group provided thoughtful and constructive comments on it and much discussion emerged from the deep and considered critique of the content. For example, group members (service and community representatives) together expressed concern about a section of the draft where grief had been (inadvertently) described in a medicalized and pathologized manner without adequate contextualization or regard to alternative models and approaches. These members did not hesitate to express their disapproval and provide alternate wording. Other examples included the group members' questions over the proposed funding model for the service, requests that the report include more detail on the effects of traffic crash injuries and the out-of-pocket costs that are borne by families following traffic crashes, suggestions regarding terminology (e.g., that 'carer' or 'unpaid family caregiver' were preferable to 'caregiver') and formatting (e.g., the use of a table to summarize the strengths and limitations of road trauma support services elsewhere in Australia), and the incorporation of references to state and national road safety strategies.

We believe this progression from consultation to participation was because the members' participation was meaningful and they felt supported, trusted, valued [40]. While the reference group did not control or direct all aspects of the study, the group had considerable influence over shaping the agenda of the study, the methods used, and the recommendations that were proposed. Ultimately, the participation achieved was extremely positive and bodes well for future research, given the challenges described earlier.

To our knowledge, this is the first project to draw upon CBPR in road trauma research. The use of CBPR in this project demonstrated some of the strengths and challenges of the methodology in developing a community-based research partnership. The development of this partnership facilitated the creation of a space for people with different experiences, opinions, and backgrounds to come together [38], because of their shared interests in road trauma issues, and to effect change. To date, the community-based research partnership has resulted in a change in government policy and practice, with the Government of Western Australia announcing in May 2012 it would fund a service according to the recommendations outlined in the report. The service, Road Trauma Support Western Australia, was launched in November 2013 on the World Day of Remembrance for Road Traffic Victims. Many of the project's group members are members of the service's steering group and this is indicative of the way in which the group has continued to have ownership of the project beyond the research phase. The diversity and depth of the stakeholder reference group - its members including representatives from relevant services as well as people affected personally by road trauma - enhances the study's ability to contribute to policy and practice [41]. Further, the report will provide the basis for the ongoing development and evaluation of the newly-established service.

\section{Conclusions}

Although it is increasingly common for stakeholders to have a role in decisions that affect them, the involvement can often be tokenistic [37] and the action aims tend to be are easier to achieve than the emancipatory aims [42]. Indeed, participatory approaches can be paradoxically used (and abused) to silence debate and defuse conflict between the powerful and powerless [43]. For instance, in health research, there is often one token consumer or one articulate consumer, leading to major 
power differentials between them and the recognized 'experts' in the committee, group, or service $[44,45]$. The research project shows that elements of CBPR can be incorporated into public health research, even in projects with externally-imposed time and budget constraints. Clearly, it is the ways in which the participation is facilitated that matters.

In describing a participatory research project that resulted in changes to health policy and practice, this paper contributes to the current discussion concerning the extent of participation in participatory research aiming to address public health concerns. Often, a critical analysis of participation within the research process is glossed over in describing research outcomes whereas in this paper there is an emphasis on the complexities, limitations, and possibilities of participatory research. We highlighted the dynamic interplay of factors that hinder and enable participation in participatory research. These reflections are useful in determining the strengths and limitations of this particular study and may also be useful to others interested in using CBPR for public health outcomes. A key advantage of this design was that it afforded the stakeholders the opportunity to generate meaningful knowledge and sustained action for their community. As a methodology at the intersection of science and practice, CBPR is likely to be an important tool for an action-oriented and community-driven public health.

\section{Competing interests}

The authors declare that they have no competing interest.

\section{Authors' contributions}

LB conceived of the study, facilitated the data collection and analysis and drafted the manuscript. MO participated in the running of the study and helped to draft the manuscript. Both authors read and approved the final manuscript.

\section{Acknowledgements}

The study was funded by the Department of Health (Western Australia) and the first author is supported by the Australian Research Council (DE120101640). The funding bodies had no involvement in the design, collection, analysis, and interpretation of data; in the writing of the manuscript; or in the decision to submit the manuscript for publication. We thank the stakeholder reference group for their expertise in directing the study.

Received: 6 November 2014 Accepted: 12 December 2014 Published: 19 December 2014

\section{References}

1. World Health Organization: Global Status Report on Road Safety 2013: Supporting a Decade of Action. Geneva: 2013.

2. Lozano R, Naghari M, Foreman K, Lim S, Shibuya K, Aboyans V, Abraham J, Adair T, Aggarwal R, Ahn SY, Alvarado M, Anderson HR, Anderson LM, Andrews KG, Atkinson C, Baddour LM, Barker-Collo S, Bartels DH, Bell ML, Benjamin EJ, Bennett D, Bhalla K, Bikbov B, Bin Abdulhak A, Birbeck G, Blyth F, Bolliger I, Boufous S, Bucello C, Burch M, et al: Global and regional mortality from 235 causes of death for 20 age groups in 1990 and 2010: A systematic analysis for the Global Burden of Disease Study 2010. Lancet 2012, 380:2095-2128.

3. World Health Organization: World Report on Road Traffic Injury Prevention Geneva: 2004
4. Ameratunga SN, Norton RN, Bennett DA, Jackson RT: Risk of disability due to car crashes: a review of the literature and methodological issues. Injury 2004, 35:1116-1127.

5. Singh R, Singh HK, Gupta SC, Kumar Y: Pattern, severity and circumstances of injuries sustained in road traffic accidents: a tertiary care hospital-based study. Indian J Comm Med 2014, 39:30-34.

6. Murphy SA, Johnson LC, Wu L, Fan JJ, Lohan J: Bereaved parents' outcomes 4 to 60 months after their children's death by accident suicide, or homicide: a comparative study demonstrating differences. Death Stud 2003, 27:39-61.

7. Rassool SB, Nel PW: Experiences of causing an accidental death: an interpretative phenomenological analysis. Death Stud 2012, 36:832-857.

8. Arnberg FK, Rydelius PA, Lundin T: A longitudinal follow-up of posttraumatic stress: from 9 months to 20 years after a major road traffic accident. Child Adolesc Psychiatry Ment Health 2011, 5:8.

9. Hours M, Chossegros L, Charnay P, Tardya H, Nhac-Vua H-T, Boisson D, Luauté J, Laumon B: Outcomes one year after a road accident: results from the ESPARR cohort. Accid Anal Prev 2013, 50:92-102.

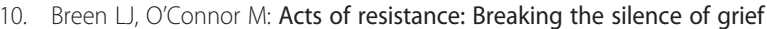
following traffic crash fatalities. Death Stud 2010, 34:30-53.

11. Breen $\sqcup, \mathrm{O}^{\prime}$ Connor M: Family and social networks after bereavement: Experiences of support, change, and isolation. J Fam Ther 2011, 33:98-120.

12. Lucke KT, Coccia H, Goode JS, Lucke JF: Quality of life in spinal cord injured individuals and their caregivers during the initial 6 months following rehabilitation. Qual Life Res 2004, 13:97-110.

13. Hoang HT, Pham TL, Vo TT, Nguyen PK, Doran CM, Hill PS: The costs of traumatic brain injury due to motorcycle accidents in Hanoi, Vietnam. Cost Eff Resour Alloc 2008, 6:17.

14. Marsh NV, Kersel DA, Havill JH, Sleigh JW: Caregiver burden during the year following severe traumatic brain injury. J Clin Exp Neuropsychol 2002, 24:434-447.

15. Connelly $L B$, Supangan $R$ : The economic costs of road traffic crashes: Australia, states and territories. Accid Anal Prev 2006, 38:1087-1093.

16. Lower T, Pollock K, Herde E: Australian quad bike fatalities: what is the economic cost? Aust N Z J Public Health 2013, 37:173-178.

17. Ameratunga $S$, Hijar M, Norton R: Road-traffic injuries: confronting disparities to address a global-health problem. Lancet 2006, 367:1533-1540.

18. World Health Organization: Advocating for Road Safety and Road Traffic Injury Victims: A Guide for Nongovernmental Organizations. Geneva: 2012.

19. Minkler M: Community-based research partnerships: challenges and opportunities. J Urban Health 2005, 82:ii53-ii62.

20. Campbell C, Murray M: Community health psychology: promoting analysis and action for social change. J Health Psychol 2004, 9:187-195.

21. Friedman DB, Young VM, Freedman DA, Adams SA, Brandt HM, Xirasagar S, Felder TM, Ureda JR, Hurley T, Khang L, Campbell D, Hébert JR: Reducing cancer disparities through innovative partnerships: a collaboration of the South Carolina Cancer Prevention and Control Research Network and federally qualified health centers. J Cancer Educ 2012, 27:59-61.

22. Gebbie K, Rosenstock L, Hernandez LM: Who will keep the public healthy? Educating public health professionals for the $21^{\text {st }}$ century. Institute of Medicine: Washington, DC; 2002.

23. Israel BA, Eng E, Schulz AJ (Eds): Methods for Community-based Participatory Research for health. 2nd edition. Somerset, NJ: Wiley; 2012

24. Minkler M, Wallerstein N: Community-based Participatory Research for Health: From Process to Outcomes. 2nd edition. San Francisco: Jossey-Bass; 2008.

25. Israel BA, Schulz AJ, Parker EA, Becker AB: Review of community-based research: assessing partnership approaches to improve public health. Annu Rev Public Health 1998, 19:173-202.

26. Nelson G, Ochocka J, Griffin K, Lord J: "Nothing about me, without me": participatory action research with self-help/mutual-aid organizations for psychiatric consumers/survivors. Am J Community Psychol 1998, 26:881-912.

27. Duckett PS, Fryer D: Developing empowering research practices with people who have learning disabilities. J Community Appl Soc Psychol 1998, 8:57-65.

28. Fine M, Torre ME: Intimate details: participatory action research in prison. Action Res 2006, 4:253-269.

29. Mirza M, Gossett A, Chan N, Burford L, Hammel J: Community reintegration for people with psychiatric disabilities: challenging systemic barriers to service provision and public policy through participatory action research. Disabil Soc 2008, 23:323-336. 
30. Morsillo J, Prilleltensky I: Social action with youth: interventions, evaluation, and psychopolitical validity. J Community Psychol 2007, 35:725-740

31. Baum F, MacDougall C, Smith D: Participatory action research. J Epidemiol Community Health 2006, 60:854-857.

32. Arieli D, Friedman VJ, Agbaria K: The paradox of participation in action research. Action Res 2009, 7:263-290.

33. Ponic $P$, Frisby $W$ : Unpacking assumptions about inclusion in communitybased health promotion: perspectives of women living in poverty. Qual Health Res 2010, 20:1519-1531.

34. Australian Transport Council: National Road Safety Strategy 2011-2020. Canberra: 2011.

35. Breen LJ, O'Connor M, Le AT, Clarke J: Establishing a Sustainable Road Trauma Support Service in Western Australia. Perth, Australia: Curtin University; 2011.

36. Breen LJ, O'Connor M, Le AT: Establishing a Sustainable Road Trauma Support Service in Western Australia, Proceedings of the 12th Australasian College of Road Safety conference (peer-reviewed). ; 2012. Available at http://acrs.org.au/files/papers/38_Breen-PR.pdf.

37. Duckett P, Kagan C, Sixsmith J: Consultation and participation with children in healthy schools: choice, conflict and context. Am J Community Psychol 2010, 46:167-178.

38. Kagan C, Duggan K: Creating community cohesion: the power of using innovative methods to facilitate engagement and genuine partnership. Soc Pol Soc 2011, 10:393-404

39. Radermacher $\mathrm{H}$, Sonn $\mathrm{C}$ : Towards getting it right: participatory action research (PAR) with an advocacy organisation. Aust Community Psychol 2007, 19(1):62-73.

40. Radermacher H, Sonn C, Keys C, Duckett P: Disability and participation: it's about us but still without us! I Community Appl Soc Psychol 2010, 20:333-346.

41. Daly J, Willis K, Small R, Green J, Welch N, Kealy M, Hughes E: A hierarchy of evidence for assessing qualitative health research. J Clin Epidemiol 2007, 60:43-49.

42. Bradbury-Huang $\mathrm{H}$ : What is good action research? Why the resurgent interest? Action Res 2010, 8:93-109.

43. Banister P, Burman E, Parker I, Taylor M, Tindall C: Qualitative methods in psychology: A research guide. Buckingham, UK: Open University Press; 1994.

44. Church K: Beyond "bad manners": the power relations of "consumer participation" in Ontario's community mental health system. Can J Community Ment Health 1996, 15(2):27-44.

45. Nelson G, Lord J, Ochocka J: Empowerment and mental health in community: narratives of psychiatric consumer/survivors. J Community Appl Soc Psychol 2001, 11:125-142.

doi:10.1186/1756-0500-7-936

Cite this article as: Breen and $\mathrm{O}^{\prime} \mathrm{Connor:} \mathrm{From} \mathrm{consultation} \mathrm{to} \mathrm{participation}$ in public health research: reflections on a community-based research partnership. BMC Research Notes 2014 7:936.

\section{Submit your next manuscript to BioMed Central and take full advantage of:}

- Convenient online submission

- Thorough peer review

- No space constraints or color figure charges

- Immediate publication on acceptance

- Inclusion in PubMed, CAS, Scopus and Google Scholar

- Research which is freely available for redistribution 